\title{
Gelatine sponge application in myoma bed to achieve hemostasis during myomectomy - A pilot study
}

\author{
Manidip Pal', Suvanjan Chatterjee ${ }^{2}$, Soma Bandyopadhyay ${ }^{3}$ \\ ${ }^{1}$ Associate Professor, Department of Obstetrics \& Gynecology, College of Medicine \& JNM Hospital, WBUHS, Kalyani, \\ West Bengal, India, ${ }^{2}$ Clinical Tutor, Department of Anesthesiology, College of Medicine \& JNM Hospital, WBUHS, \\ Kalyani, West Bengal, India, ${ }^{3}$ Professor, Department of Obstetrics \& Gynecology, Katihar Medical College, Bihar, India
}

Aims and Objectives: To study the efficacy of gelatine absorbable sponge to achieve hemostasis of myoma bed during myomectomy. Materials and Methods: Patient $<40$ years with diagnosis of fibroid planned for myomectomy were included. Local infiltration around myoma was done with vasopressin diluted with normal saline (10 units of vasopressin mixed with $100 \mathrm{ml}$ of normal saline). Myomas are easily removed. Gelatin absorbable sponge was applied over the myoma bed and the bed was closed. Proper hemostasis was maintained. Hemoglobin deficit was measured postoperatively. Results: Fifteen cases were studied. Vasopressin (diluted) administration ranged from 30-200 ml. Intra-operative blood loss of about $50 \mathrm{ml}$ was noticed in 6 cases, $80 \mathrm{ml}-3$ cases, $100 \mathrm{ml}-3$ cases, $200 \mathrm{ml}$ - 2 cases, $400 \mathrm{ml} \mathrm{-} 1$ case. Duration of operation ranged from 60 - 150 minutes. Pre and post-operative $\mathrm{Hb} \%$ estimation revealed that there was no change in $\mathrm{Hb} \%$ in 3 cases. Conclusion: Gelatin absorbable sponge application over the myoma bed while closing the area is a good technique to achieve hemostasis during myomectomy.

Key words: Hemorrhage, Intraoperative, Vasopressin

\section{INTRODUCTION}

Uterine fibroid or myoma is a very common gynaecological disease. Reported incidence is about $25 \%$ irrespective of the age. ${ }^{1}$ In premenopausal women it accounts for about $30-70 \%{ }^{2}$ It causes different types of menstrual problems (e.g. menorrhagia, metrorrhagia, dysmenorrhoea etc.), pressure symptoms, bloated sensation, increased urinary frequency, bowel disturbance, or pelvic pain etc. ${ }^{3}$ Fibroid could be a cause of sub-fertility also. Fibroid detected in menopausal women usually managed conservatively thinking on the fact that after menopause due to the low estrogen level fibroid shrinks in size. In young women removal of the fibroid is to be done, though nowadays fibroids can be resolved by nonsurgical techniques also. Embolization of the uterine artery, ${ }^{4,5}$ ultrasonic resolution of the fibroids by MRgFUS (Magnetic resonance guided focused ultrasound), ${ }^{6,7}$ are some conservative approaches to myoma. But these facilities are not available everywhere. Depending on the size and site of the fibroid, surgical technique of myoma removal varies. Submucosal fibroid is usually removed by hysteroscopic resection. Intramural and subserous fibroid can be removed by laparotomy or laparoscopy or robotic surgery. Bleeding from the myoma bed after myoma removal is one of the great operative problems. To overcome this problem, many techniques have been adopted since years. The objective of the present study is to evaluate a technique to prevent blood loss from the myoma bed during myomectomy.

\section{MATERIALS AND METHODS}

Patients attending gynecology OPD with the diagnosis of fibroid were included. The study was conducted from April 2015 to Nov 2015. Inclusion criteria were - younger 
patient (age less than 40 years), wants to preserve her fertility and menstrual capability. Subserous (not pedunculated) and intramural fibroids were included. Exclusion criteria - patient's with known allergies to porcine collagen. Proper history was taken along with their presenting complaints e.g. menstrual irregularities, pressure symptoms etc. General physical examination and gynaecological examination were done. Routine preoperative investigations were done. Ultrasonography was done to map the fibroids. If the patients were anaemic then hemoglobin was raised to minimum $10 \mathrm{gm} \%$ before the operation. Blood requisition was done for intraoperative SOS use. Informed consent was obtained from the patient. Consent for hysterectomy, if situation demand, to save the patient's life, was also taken - though none of the patient needed hysterectomy. This study was approved by the Institutional Ethics Committee of College of Medicine \& JNM Hospital, WBUHS, Kalyani, West Bengal, India.

Operations were done by laparotomy. Location of the myoma(s) was found out. Uterus made steady by the assistant. Vasopressin injection $1 \mathrm{ml}$ (20 units) was diluted with $200 \mathrm{ml}$ normal saline. Guideline was 10 units for $100 \mathrm{ml}$ of normal saline. ${ }^{8}$ Now depending on number and size of fibroids diluted preparation was injected. Solution was injected around the periphery of the fibroid. Care was taken to complete the infiltration in one area by one needle prick, if possible. Blanching of the fibroid could be seen. Incision was made over the fibroid and it was made detached from its surrounding through its capsule (Photo 1,4). Now closure of the myoma bed is started. A bite at one corner of the bed, incorporating the tissue upto the bottom of the bed, was taken and tied. An absorbable gelatine sponge (AbGel, Shri Gopal Krishna Labs Pvt. Ltd., Mumbai, India) was placed at the myoma bed (Figs. 1-3; Photo 2).

Gelatine absorbable sponge is a water-insoluble, off-white, nonelastic, porous, pliable product prepared from purified porcine skin (Gelatin USP Granules). It is been used to control bleeding from tissue surfaces. It is non-toxic, non-pyrogenic, non-allergic, non-immunogenic. Gelatine forms an artificial clot, a mechanical matrix that facilitates clotting. Sponge after application over the bleeding surfaces absorbed platelets. These platelets get damaged, once they come in contact with the walls of myriad of interstices of sponge. As a result thromboplastin gets released from platelets. This thromboplastin initiates the coagulation cascades and clot formed. The spongy physical properties of the gelatine sponge hasten clot formation and provide structural support for the forming clot. Sponge is completely absorbed in 4-6 weeks.

Depending on the size of the myoma bed, the sponge size was customized. Again bite by the previous suture was taken in same manner i.e. incorporating the bottom tissue of the myoma bed. That means the suture was then encircling the gelatine sponge along with the myoma bed tissue (Fig. 4). Suture pulled in an effort to obliterate the myoma bed. Like that way suture continued till the other corner of the myoma bed and then knot applied (Fig. 5). It was a continuous simple suture. In that way myoma bed dead-space was obliterated along with the hemostatic gelatine sponge within (Fig. 6, Photo 3). Dual effect of mechanical compression by suture and local gelatine helped in achieving hemostasis. If the depth of the myoma bed was more, then more layers of sutures were placed. Once the cavity was obliterated, the serosal margin was closed by baseball continuous suture so as to invert the margin. This would prevent adhesion formation. If multiple myomas were present and if they could be approached through one serosal incision, then they were removed through that. After securing proper hemostasis, peritoneal toileting was done with normal saline. Abdomen closed in layers. Before closing Inj. Dexamethasone $20 \mathrm{mg}$ was instilled inside the peritoneal cavity. Same dose was given intramuscularly 2 hours before the surgery. Postoperatively Inj Dexamethasone $20 \mathrm{mg}$ was given intramuscularly every 4 hours X 12 doses. First dose was given 4 hours after the operation. This is also to prevent adhesion formation. ${ }^{10}$ Exact estimation of blood loss could not be measured. Approximate figure was calculated by subjective assessment of swabs, gauze pieces and amount in suction bottle. Postoperative complication if any (e.g. bleeding, tachycardia, hypotension etc.) were noted.

\section{RESULTS}

Age of the patient ranged from 28 -37 years. Out of the 15 cases, solitary fibroid was present in 6 cases. In multiple fibroids, there was mix up of intramural and subserous fibroids. Amount of diluted vasopressin administered ranged from 30-200 ml; most of the patient needed 50-70 ml. Six patient had intra-operative blood loss about $50 \mathrm{ml}$, only 1 patient had blood loss about $400 \mathrm{ml}$. Three patients received blood transfusion. Duration of operation ranged from 60 - 150 minutes; in 6 cases time required was 90 minutes. Hemoglobin changes from preoperative to postoperative revealed unchanged in 3 cases. Interesting finding was that when the learning curve advanced, the operator became more conversant with the procedure which was reflected in the second case of 24 weeks intramural solitary fibroid, where they used $100 \mathrm{ml}$ diluted vasopressin with blood loss about and operative time was 90 minutes.

\section{DISCUSSION}

Myomectomy is an age-old operation. But due to intraoperative torrential bleeding possibilities, it is a great 


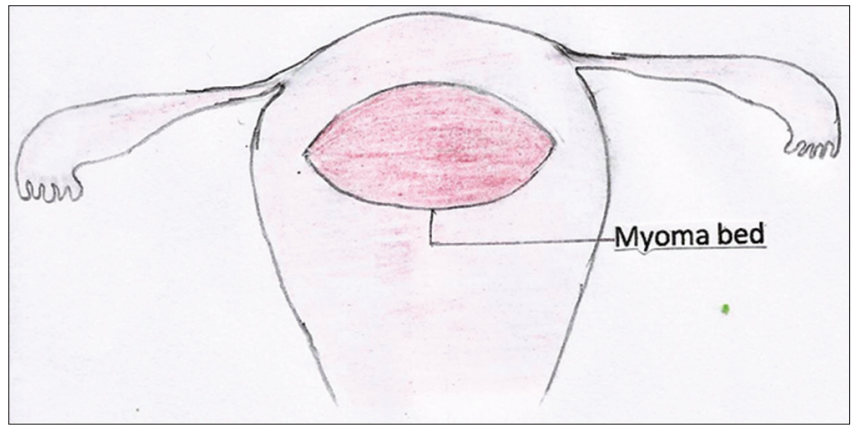

Figure 1: Myoma bed after removal of myoma from posterior uterine wall

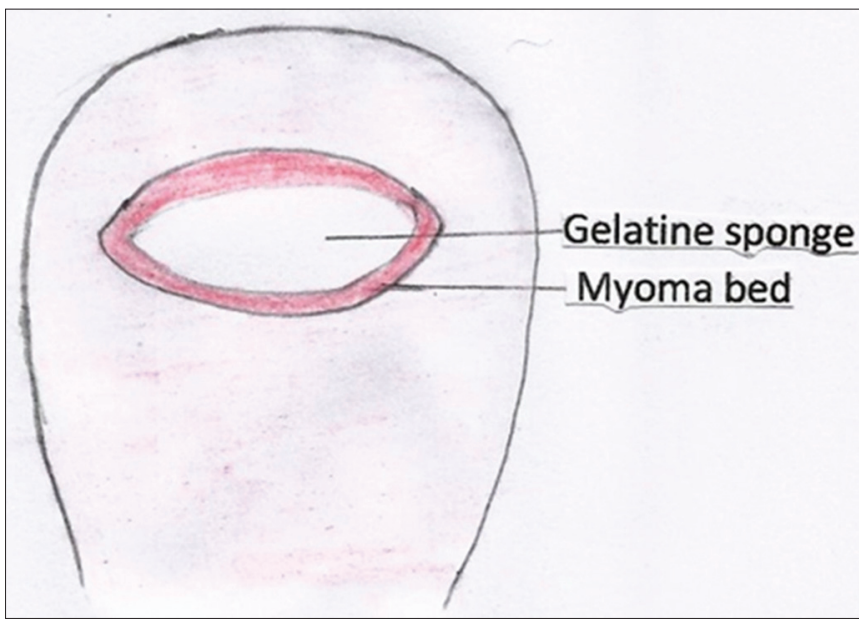

Figure 2: Gelatin absorbable sponge (white colored) applied directly over the myoma bed

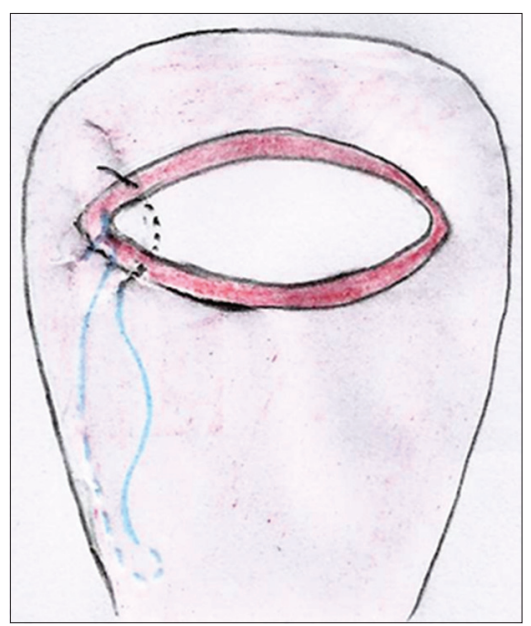

Figure 3: Needle bite at myoma bed is taken to close the myoma bed. Starting point of the needle is within the side-wall of the myoma bed just little below the serosal margin. Traversing just below the undersurface of the bed and then coming out again through the side-wall of the myoma bed just little below the serosal margin

concern to the surgeon always. Different techniques have been applied to minimise this complication. Local administration of vasopressin has kept the operative field blood free. Hence meticulous suturing was possible. Diluted

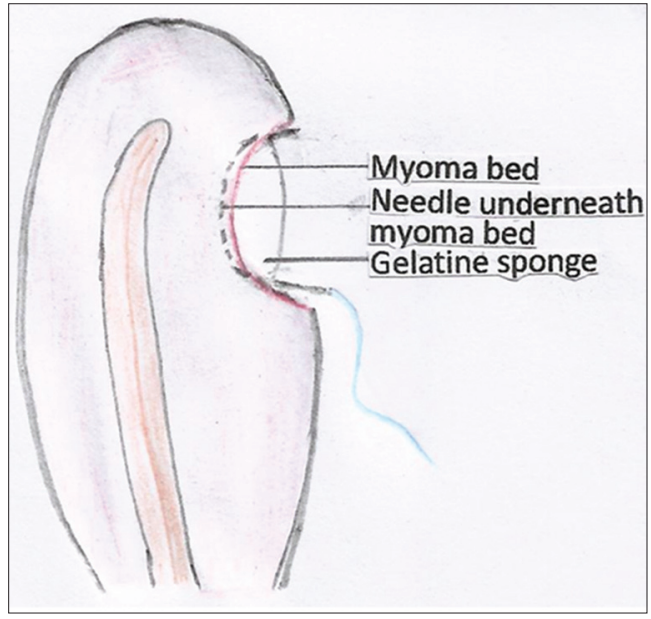

Figure 4: Sagittal view of the myoma bed showing how the needle is passing just below the undersurface of bed. Gelatin sponge-in-situ

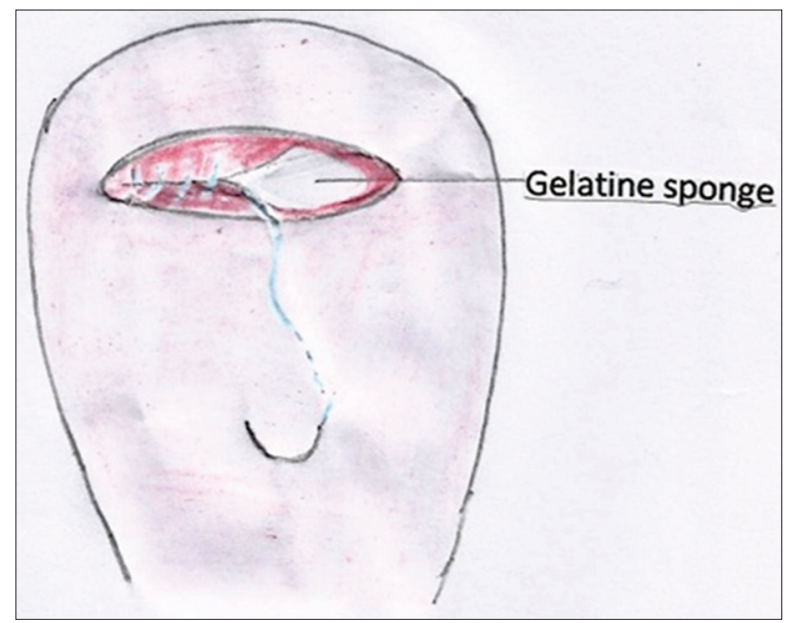

Figure 5: Half of the myoma bed is closed - it is a continuous simple suturing. Gelatin sponge inside can be seen

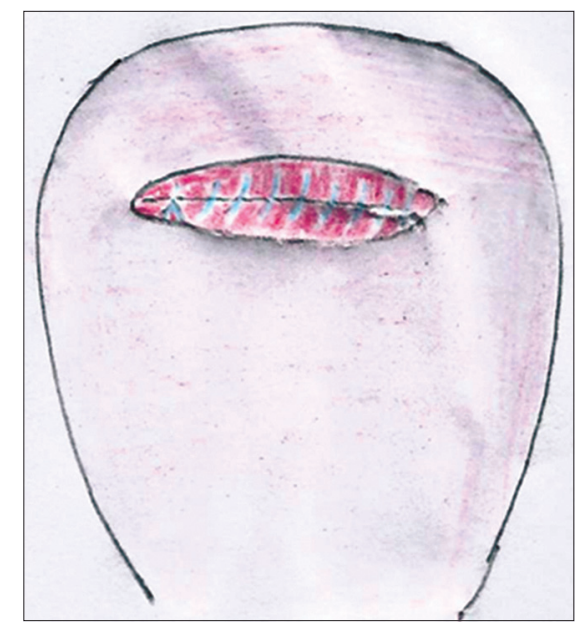

Figure 6: Whole of the myoma bed is closed. Next, serosal margin of the bed will be closed by continuous baseball suturing

vasopressin local infiltration was used to have a clear blood less operative field. In a randomized placebo controlled trial 


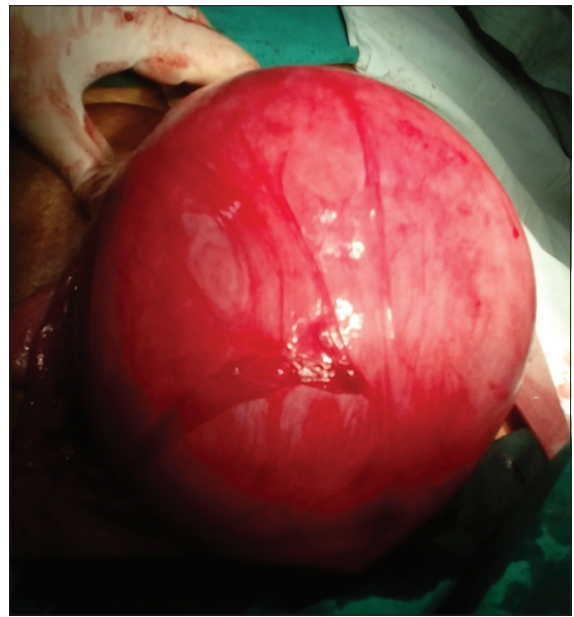

Photo 1: Solitary intramural myoma 24 weeks size

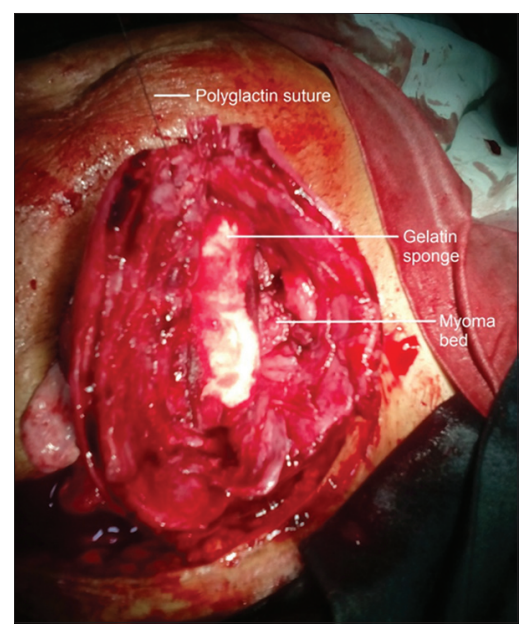

Photo 2: Myoma removed $\rightarrow$ closure of the myoma bed was started from the angle of the bed with polyglactin suture $\rightarrow$ absorbable gelatine sponge was applied at the bottom of the myoma bed

of myomectomy, vasopressin 20 units diluted with $20 \mathrm{ml}$ normal saline was injected intramyometrially. Median blood loss in vasopressin group was $225 \mathrm{ml}$ in compare to $675 \mathrm{ml}$ in placebo group. ${ }^{11}$ Diluted vasopressin injection around the myoma is a simple, effective, and safe homeostatic procedure during myomectomy. ${ }^{12}$ Other hemostatic agents use are bupivacaine with epinephrine, oxytocin, tranexamic acid etc. In a Cochrane review ${ }^{13}$ different interventions used to reduce bleeding during myomectomy was assessed. The interventions were intramyometrial vasopressin (two RCTs), intravenous oxytocin (two RCTs), peri-cervical tourniquet (two RCTs), and one RCT each for vaginal misoprostol, gelatine-thrombin matrix, chemical dissection with sodium-2-mercaptoethane sulfonate (mesna), intramyometrial bupivacaine plus epinephrine, tranexamic acid, and myoma enucleation by morcellation. Findings were significant reductions in blood loss with misoprostol \{Mean Difference (MD) -149.00 ml, 95\% CI -229.24 to -68.76$)\}$, vasopressin (MD $-298.72 \mathrm{ml}$,

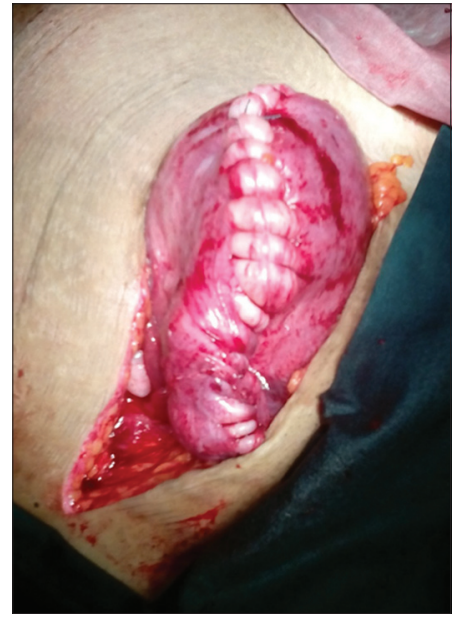

Photo 3: At the end of the closure $\rightarrow$ the serosal surface was seen $\rightarrow$ in this case the myoma bed was closed in 3 layers $\rightarrow$ in each layer absorbable gelatine sponge was applied

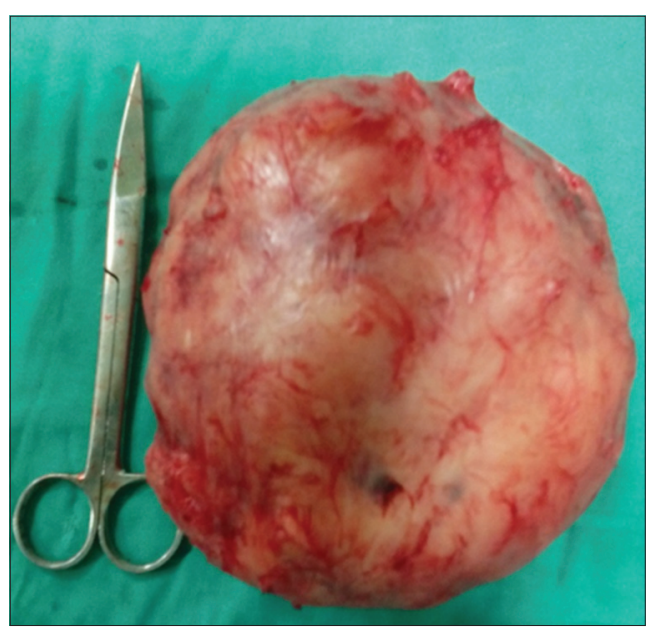

Photo 4: The myoma was almost the size of a medium scissor

95\% CI -593.10 to -4.34 ; I(2) = 99\%), bupivacaine plus epinephrine (MD -68.60 ml, 95\% CI -93.69 to - 43.51), tranexamic acid (MD $-243 \mathrm{ml}, 95 \% \mathrm{CI}-460$ to -25.98$)$, peri-cervical tourniquet (MD -289.44, 95\% CI -406.55 to $-172.32 ; \mathrm{I}(2)=95 \%$ ), and gelatine-thrombin matrix (MD -545.00 ml, 95\% CI -593.26 to -496.74). The review was concluded that evidence was limited to give final opinion about which hemostatic agent is the best.

Blood loss ranged from 20 to $250 \mathrm{ml}$. The operating time was 50-100 mins. No hemorrhagic complication occurred in any patient during surgery or postoperatively. ${ }^{8}$ In present study $6 \%$ patient had intra-operative blood loss about $50 \mathrm{ml}$. Three patients received blood transfusion. Duration of operation ranged from $60-150$ minutes.

Uterine artery embolization with gelatine sponge particles prior to myomectomy yields a good result in terms of intraopertive blood loss and improves the chances 
of performing conservative surgery. Two studies with preoperative gelatine sponge embolization were conducted on $21^{14} \& 33^{15}$ patients - results were good. One study was conducted to see the prospect of gelatine sponge embolization (11 cases) of myoma as minimally invasive treatment of leiomyoma. It was compared with traditional particle embolization. But gelatine embolization yields poor results in terms of purulent necrosis in compare to traditional particles embolization. ${ }^{16}$ A systematic review was done on use of gelatine-thrombin matrix for intraoperative hemostasis in different abdomino-pelvic surgery and regarding open myomectomy it was found that gelatinethrombin matrix dramatically reduced intraoperative bleeding and transfusion rates. ${ }^{17}$ In present study gelatine sponge was applied directly over the bleeding myoma bed to obtain hemostasis and results were good.

Drawback of this study is that the study is small and does not have control group with conventional sutures. Hence final comment could not be made. As the results are promising, we wish to conduct further study with more number of patients and control group.

\section{CONCLUSION}

Gelatine sponge over the myoma bed achieves good local hemostasis. Diluted vasopressin aqua-dissection eases the operative procedure more.

\section{REFERENCES}

1. Biglia N, Carinelli S, Maiorana A, D'Alonzo M, Lo Monte G and Marci R. Ulipristal acetate: a novel pharmacological approach for the treatment of uterine fibroids. Drug Des Devel Ther 2014; 8:285-292.

2. Marshall LM, Spiegelman $D$, Barbieri RL, Goldman MB, Manson JE, Colditz GA, et al. Variation in the incidence of uterine leiomyoma among premenopausal women by age and race. Obstet Gynecol 1997; 90(6): 967-973.

3. Horng HC, Wen KC, Su WH, Chen CS and Wang PH. Review of myomectomy. Taiwan J Obstet Gynecol 2012; 51(1): 7-11.

4. Arthur R, Kachura J, Liu G, Chan C and Shapiro H. Laparoscopic myomectomy versus uterine artery embolization: long-term impact on markers of ovarian reserve. J Obstet Gynaecol Can 2014; 36(3): 240-247.

5. Torre A, Paillusson B, Fain V, Labauge P, Pelage JP and Fauconnier A. Uterine artery embolization for severe symptomatic fibroids: effects on fertility and symptoms. Hum Reprod 2014; 29(3): 490-501.

6. Schlesinger D, Benedict S, Diederich C, Gedroyc W, Klibanov A and Larner J. MR-guided focused ultrasound surgery, present and future. Med Phys 2013; 40(8): 080901.

7. Desai SB, Patil AA, Nikam R, Desai AS and Bachhav V. Magnetic resonance-guided focused ultrasound treatment for uterine fibroids: first study in Indian women. J Clin Imaging Sci 2012; 2(1): 74 .

8. Modi R. Laparoscopic myomectomy with aquadissection and barbed sutures. J Gynecol Endosc Surg 2011; 2(1): 47-52.

9. Available from: URL: http://www.pfizer.com/files/products/uspi_ gelfoam_plus.pdf.

10. Newton JR. Operations for the correction of infertility. In: Monaghan JM, ed. Bonney's Gynaecological Surgery. $9^{\text {th }}$ edn. East Sussex: Bailliere Tindall, 1992, pp 149-167.

11. Frederick J, Fletcher $\mathrm{H}$, Simeon $\mathrm{D}$, Mullings $\mathrm{A}$ and Hardie $\mathrm{M}$. Intramyometrial vasopressin as a haemostatic agent during myomectomy. Br J Obstet Gynaecol 1994; 101(5):435-437.

12. Lin XN, Zhang SY, Fang SH, Wang MZ and Lou HY. Assessment of different homeostatic methods used in laparoscopic intramural myomectomy. Zhonghua Yi Xue Za Zhi 2008; 88(13):905-908.

13. Kongnyuy EJ and Wiysonge CS. Interventions to reduce haemorrhage during myomectomy for fibroids. Cochrane Database Syst Rev 2011 Nov 9;(11):CD00535 5. doi: 10.1002/14651858. CD005355.pub4

14. Tixier H, Loffroy R, Filipuzzi L, Grevoul J, Mutamba W, Cercueil J, et al. Uterine artery embolization with resorbable material prior to myomectomy. J Radiol 2008; 89(12):1925-1929.

15. Butori N, Tixier H, Filipuzzi L, Mutamba W, Guiu B, Cercueil JP, et al. Interest of uterine artery embolization with gelatin sponge particles prior to myomectomy for large and/or multiple fibroids. Eur J Radiol 2011; 79(1):1-6.

16. McLucas $B$, Chespak $L$ and Kaminsky D. Myoma necrosis following Gelfoam embolization of uterine myomata. Minim Invasive Ther Allied Technol 2008; 17(3):200-204.

17. Mayol JM and Zapata C. Gelatin-thrombin matrix for intraoperative hemostasis in abdomino-pelvic surgery: a systematic review. Surg Technol Int 2013; 23:23-28.

\footnotetext{
Authors Contribution:

MP - Concept and design of the study, collected data, manuscript preparation; SC - Design of the study, collected data, manuscript preparation; SB - Concept and design of the study, manuscript preparation
}

Source of Support: Nil. Conflict of Interest: None. 